\title{
Transient global amnesia: implicit/explicit memory dissociation and PET assessment of brain perfusion and oxygen metabolism in the acute stage
}

Francis Eustache, Béatrice Desgranges, Marie-Christine Petit-Taboué, Vincent de la Sayette, Véronique Piot, Christelle Sablé, Gilles Marchal, Jean-Claude Baron

INSERM U320

F Eustache

B Desgranges

M-C Petit-Taboué

$\mathrm{V}$ de la Sayette

V Piot

C Sablé

G Marchal

J-C Baron

Services de Neurologie, Centre Hospitalier

Universitaire Côte de Nacre, University of

Caen, France

F Eustache

B Desgranges

V de la Sayette

V Piot

C Sablé

Cyceron-CEA DSV/DRM, Caen, France

M-C Petit-Taboue

G Marchal

J-C Baron

Correspondence to:

Francis Eustache, Services de Neurologie et INSERM U320, Centre Hospitalier

Universitaire Côte de Nacre, 14033 Caen Cedex, France or to

Jean-Claude Baron,

INSERM U.320, Cyceron, BP 5229, 14074, Caen,

France.

Received 8 October 1996 and in revised form

6 March 1997

Accepted 25 April 1997
Abstract

Objectives-To assess explicit memory and two components of implicit memory-that is, perceptual-verbal skill learning and lexical-semantic priming effects-as well as resting cerebral blood flow (CBF) and oxygen metabolism $\left(\mathrm{CMRO}_{2}\right)$ during the acute phase of transient global amnesia.

Methods-In a 59 year old woman, whose amnestic episode fulfilled all current criteria for transient global amnesia, a neuropsychological protocol was administered, including word learning, story recall, categorical fluency, mirror reading, and word stem completion tasks. PET was performed using the ${ }^{15} O$ steady state inhalation method, while the patient still exhibited severe anterograde amnesia and was interleaved with the cognitive tests.

Results-There was a clear cut dissociation between impaired long term episodic memory and preserved implicit memory for its two components. Categorical fluency was significantly altered, suggesting word retrieval strategy-rather than semantic memory-impairment. The PET study disclosed a reduced $\mathrm{CMRO}_{2}$ with relatively or fully preserved $\mathrm{CBF}$ in the left prefrontotemporal cortex and lentiform nucleus, and the reverse pattern over the left occipital cortex.

Conclusions-The PET alterations with patchy CBF-CMRO, uncoupling would be compatible with a migraine-like phenomenon and indicate that the isolated assessment of perfusion in transient global amnesia may be misleading. The pattern of metabolic depression, with sparing of the hippocampal area, is one among the distinct patterns of brain dysfunction that underlie the (apparently) uniform clinical presentation of transient global amnesia. The finding of a left prefrontal hypometabolism in the face of impaired episodic memory and altered verbal fluency would fit present day concepts from PET activation studies about the role of this area in episodic and semantic memory encodingl retrieval. Likewise, the changes affecting the lenticular nucleus but sparing the caudate would be consistent with the normal performance in perceptual-verbal skill learning. Finally, unaltered lexicalsemantic priming effects, despite left temporal cortex hypometabolism, suggest that these processes are subserved by a more distributed neocortical network.

(F Neurol Neurosurg Psychiatry 1997;63:357-367)

Keywords: transient global amnesia; implicit/explicit memory dissociation; oxygen metabolism; PET

Forty years after its original description, the pathophysiology of transient global amnesia remains elusive, with three major hypotheses: a transient ischaemia, a seizure process, or a migrainous phenomenon. ${ }^{12}$ Likewise, despite extensive neurological accounts, ${ }^{3}$ only 35 cases in whom formal (although usually rather limited) neuropsychological assessment was performed during the acute stage have been reported. ${ }^{2-19}$ Although these studies concur in showing that transient global amnesia is a pure syndrome of isolated memory impairment akin to the permanent amnesic syndrome (PAS), with a consistent dissociation between preserved short term but impaired long term memory (LTM), the exact profile of memory impairment in transient global amnesia remains incompletely known.

Thus only a few studies have assessed the distinct components of LTM during episodes of transient global amnesia, notably implicit memory (skill learning and priming effects). Apart from the above mentioned impairment in episodic memory, which is intrinsic to the definition of transient global amnesia, one consistent finding has been that semantic memory is preserved. ${ }^{481116}$ Although a dissociation between declarative memory and skill learning is suggested by the well known finding that patients with transient global amnesia are occasionally able to perform complex previously acquired skills, ${ }^{2021}$ preserved acquisition of new skills has been formally documented only rarely. ${ }^{2418}$ Likewise for priming effects, only perceptual priming effects have been studied $^{18} 19$ whereas lexical-semantic priming effects $^{22}$ have remained unexplored thus far.

Studies of resting cerebral perfusion and metabolism during transient global amnesia are of special interest because they may shed light not only on the pathophysiology, but also on the mechanisms of the cognitive impair- 
ment of transient global amnesia. Unfortunately, only few such studies have been possible for obvious logistical reasons. ${ }^{23}$ Only three patients have had both perfusion and metabolism assessed with PET, two reported in abstract form ${ }^{2425}$ and one as a full report. ${ }^{23}$ In the rest solely brain perfusion was assessed, by means of SPECT. Although many of these studies (performed either in the acute stage, the early recovery phase, or immediately after the episode) not unexpectedly disclosed unilateral or bilateral alterations in perfusion or metabolism in mesial temporal, ${ }^{2315-1824-26}$ or thalamic regions, ${ }^{214172327}$ several did find occasionally isolated changes in neocortical, notably frontal and temporal, regions, either unilaterally or bilaterally. ${ }^{21314172327-29}$ These unexpected imaging findings made the important suggestion that an acute impairment of episodic memory could be subtended by different localisations of synaptic dysfunction. ${ }^{223}$ Memory was concurrently investigated only in a few of the above studies as part of either a restricted, ${ }^{131517}$ or a more extensive assessment, ${ }^{23141618}$ but in all the imaging modality used was SPECT, and almost none provided a detailed interpretation in terms of the mechanisms of transient amnesia. As uncoupling between CBF and metabolism is frequent in acute brain conditions $^{30}$ including transient global amnesia, ${ }^{24}$ perfusion may not constitute a valid index of synaptic function under these circumstances, and thus SPECT may provide incomplete or even misleading information for both the pathophysiology and the neurobiological basis of memory dysfunction during transient global amnesia.

Our aims were to formally investigate both memory (notably perceptual-verbal skill learning and lexical-semantic priming effects), and resting cerebral perfusion and oxygen metabolism with PET during the acute phase of transient global amnesia, according to a specially designed prospective protocol. We anticipated that, by carrying out both types of investigations concurrently, we would gain further insight into the cognitive and physiological pattern(s) of transient global amnesia.

\section{Subjects and methods}

PATIENTS

Over a period of six months, four patients with suspected transient global amnesia were seen by us in the emergency unit of the University Hospital of Caen. Three fulfilled the operational criteria for transient global amnesia set out by Caplan $^{31}$ and modified by Hodges and Warlow, ${ }^{21}$ but in two of them, the episode was over before the tests could be completed. In the last patient, a 59 year old housewife, it was possible to perform both the neuropsychological investigation and PET during the episode; this patient forms the basis of the present article.

CONTROLS

A group of seven subjects, strictly matched to the patient in sex, age (mean (SD): 59.4 (2.8) years, and educational level was used for normative neuropsychological data, whenever these were unavailable from the literature, or for personal versions or French adaptations of published tests. Selection was made based on lack of neurological or psychiatric disorder or general cognitive impairment (as assessed with Signoret's "Batterie d'efficience cognitive" (BEC), ${ }^{32}$ in which each score has to be above 9/12 (see below)). Likewise, for the PET study, we constructed a control group made of 10 optimally healthy, unmedicated subjects of mean age matched to that of the patient (mean (SD): 56.5 (5.8) years). In both groups, the minimum educational level was equivalent to "certificat d'études primaires"-a now abandoned diploma obtained after eight years of primary education.

\section{NEUROPSYCHOLOGICAL INVESTIGATION}

Protocol

We prospectively constructed a brief modular neuropsychological protocol such that it could be administered in sequences interleaved with both the emergency medical investigations required to evaluate acute amnesia (for example, CT, ECG, EEG), and the PET study.

The major aim of this protocol was to assess separately the two components of implicit memory - that is, priming effects and skill learning (see below). In addition, explicit episodic memory and other cognitive functions were assessed with subtests of the "Batterie d'Efficience Mnésique" (BEM) ${ }^{33}$ and of the BEC respectively. For general cognitive functions we assessed mental control, visuoconstructive abilities (with the copy of three geometrical figures, two simple and a complex one), orientation, problem solving, and semantic similarities. Semantic memory was more specifically assessed by means of both categorical verbal fluency (generating as many names of animals as possible in one minute) and naming of 12 verbalisable (high frequency) line drawings. For episodic memory, we assessed (1) visual episodic memory with the immediate free recall of the Signoret's complex geometric figure and the free recall and the recognition of six verbalisable line drawings (different from the set of 12 used in the naming test); and (2) verbal episodic memory, by means of story recall and word learning tasks. In the word learning task, the order of recall (which was left without specific instruction) made it possible to calculate the primacy and recency effects, using a method modified from Tulving and Colotla $^{34}$ who consider a word to be recalled from short term memory (STM) if its presentation is separated from its recall by at most six words, and from LTM otherwise. Our modified procedure uses the subject's own verbal span instead of the fixed number six. In addition to the recency effect, STM was also assessed with the forward verbal span.

For implicit memory, we elected to restrict our time constrained protocol to the study of perceptual-verbal procedural memory and lexical-semantic priming effects, using modified versions of the mirror reading and the word stem completion paradigms respectively.

The mirror reading paradigm was adapted from Cohen and Squire. ${ }^{35}$ The mirror words were presented in 20 pairs in a 20 page 
notebook. The order of presentation of the words in the notebook took into account the number of b, p, q, d, and g letters, which are the most difficult in mirror reading. Firstly, two easy examples were presented to train the subjects, who were then required to read each pair of mirror words as quickly as possible. Performances were assessed according to the time taken to complete each trial. In case of failure, the maximum allotted time (90 seconds) was counted. The entire procedure was repeated an hour and a half later with different words. The data from both sessions were subjected to an original analysis which capitalised on the large number of trials and accounted for the exponential-like trend for improvement in performance. This analysis effected a linear regression on the log transformed data, from each session, which then allowed us to examine the effect of learning in each session by comparing the mean slope of each regression line obtained across the seven control subjects with zero. Likewise, retention of the mirror reading skill from session I to session II across subjects was assessed by comparing the formula (B-A)/(B+A) with zero, where $A$ and $B$ are the $y$ intercepts of the regression lines for sessions I and II, respectively.

Lexical-semantic priming effects were assessed with a modified word-stem completion paradigm. ${ }^{36}$ In the first part (study phase), and without having been instructed to memorise, the subject was required to make a sentence from each word of a series of 15 written words (of which the 10 middle words were later used for assessment of priming effects; the first two and last three being present only to reduce primacy and recency effects). During the second part (test phase), given immediately after the first, 20 written three letter stems were presented to the subject, who had to complete them orally with the first word that came to mind. Ten of the stems were the beginning of the primed words and 10 were stems of distractor words. The words were taken from a French words frequency directory, ${ }^{37}$ so as to match the primed words and the distractors for both frequency (relative frequency: 100 to 17000 occurrences per 100 million) and length (six to eight letters), and in such a way that each stem (both primed and distractors) could give rise to at least 10 common words. The priming effect shows itself during stem completion by a preferential reproduction of primed as compared to non-primed words (which may match distractors only by chance). To weight the processing towards the lexicalsemantic (and away from the perceptual) component, we purposely reduced the degree of perceptive similarity between the primed words and the stems, by using a different case form for the words and for the stems, and we induced a semantic processing of the words (constructing a sentence) during the study phase. The priming effect was expressed as the number of primed words minus the number of non-primed words that matched the selected distractors.
STATISTICAL ANALYSIS

The patient's performance in each subtest of Signoret's batteries (the BEC and the BEM) was assessed against their published normative data, when available; for each subtest, a $z$ score was calculated and judged significant if beyond the one tailed $t$ value for $\mathrm{P}<0.05$ corresponding to the sample of control subjects for each test-that is, 1.70 for the $\mathrm{BEC}$ ( $\mathrm{n}=30$ subjects) and 1.72 for the BEM ( $n=21$ subjects). For the scores obtained in the subtests of the BEM and the BEC for which no normative data are available (namely, forward verbal span, primacy, and recency effects) or the subtests for which we used personal adaptations (copy of complex geometric figure), as well as for the word stem completion and mirror reading tasks, we used as normative data the performances of our group of seven age matched and education matched controls; statistical assessment was as above, except that the $t$ value was 1.94 $(\mathrm{P}<0.05, \mathrm{n}=7)$. For the mirror reading task, the patient's slopes and retention index were compared with the control subjects according to the same general statistical procedure.

\section{PET INVESTIGATION}

\section{PET imaging}

The PET study was done according to a standardised procedure. ${ }^{38}$ We used the ${ }^{15} \mathrm{O}$ steady state inhalation method ${ }^{39}$ with serial arterial ${ }^{15} \mathrm{O}$ counting and transmission attenuation correction. We used a high resolution, 7 slice tomograph (TTV03, LETI, Grenoble, France),${ }^{40}$ with final apparent image resolution $\sim 11 \times 11 \times 9 \mathrm{~mm}$, xyz. We used the stereotaxic procedure derived from Fox et $a l^{41}$ based on the glabella and the inion bony landmarks. The seven PET planes were made parallel to the glabella-inion line and lay from $-4 \mathrm{~mm}$ to +68 $\mathrm{mm}$ relative to it. To restrain the head during scanning, we used a Laitinen stereotaxic frame. Furthermore, head position during scanning was continuously monitored and any displacement immediately corrected, by laser beams projected on forehead ink marks. The raw ${ }^{15} \mathrm{O}_{2}$ and $\mathrm{C}^{15} \mathrm{O}_{2}$ images were systematically realigned with the dedicated software of Woods et al. ${ }^{42} \mathrm{We}$ obtained parametric images of cerebral blood flow (CBF; ml/100 g/min), oxygen extraction fraction (OEF, dimensionless), and cerebral metabolic rate of oxygen $\left(\mathrm{CMRO}_{2}, \mathrm{ml} / 100\right.$ / $\mathrm{min}$ ), corrected for intravascular radiotracer, ${ }^{43}$ by applying to the raw PET images, pixel by pixel, a set of non-linear equations. ${ }^{39} 43$

\section{Regions of interest (ROI) procedure}

A three dimensional MRI (made of 128 contiguous $\mathrm{T} 1$ weighted scans) was performed one week after the PET (GE Advantage 1.5 T), according to the SPGR procedure. By means of dedicated software ${ }^{44}$ enabling MRI data acquired in three dimensions to be resliced to the PET coordinates with respect to the glabellainion line landmarks (which has known relations with the anterior commissure-posterior commissure reference points ${ }^{41}$ ), circular regions of interest (ROIs; $n=103$, radius $=7 \mathrm{~mm}$ ) were positioned directly over the MRI cross sections corresponding to the PET planes, 
according to Talairach's stereotaxic atlas, ${ }^{45}$ to sample neocortical, limbic (especially hippocampal), subcortical (namely, caudate nucleus, lentiform nucleus, and thalamus,) and cerebellar areas. ${ }^{38}$ These ROIs were projected on to corresponding PET matrices, and the ROI pixel values were averaged according to anatomical-functional cortical and noncortical areas to yield regional values for $\mathrm{CBF}$, $\mathrm{OEF}$, and $\mathrm{CMRO}_{2} \cdot{ }^{38}$ (Right-left percentage differences for homologous regions (asymmetry indices) were also calculated.)

\section{Statistical assessment}

We compared the regional index values and the (R-L) asymmetry indices obtained for each brain area in our patient to single subject confidence limits (mean (SD) $t_{\mathrm{p}}, \mathrm{df}_{\mathrm{d}}$ ) calculated from data obtained with the same methodology in 10 healthy volunteers of similar mean age. Because of the different number of ROIs included in a given anatomical-functional area (for example, from 2 for the thalamus to 14 for the lateral-frontal cortex), the accuracy in regional index estimation varied and the confidence intervals differed widely from one region to another. In addition, the confidence limits for the (R-L) asymmetry indices could be weighted towards the right or the left side due to underlying overall asymmetries in controls.

\section{Results}

Figure 1 shows the time course of the different events related to the transient global amnesia episode.

\section{CASE HISTORY}

The patient is a $100 \%$ right handed 59 year old woman with a history of treated arterial hypertension, but otherwise healthy. There was no histology of migraine.
On 16 June 1994, at 745 am, her husband was awakened by noises of retching from his wife, who also complained of headache. She asked her husband repeatedly about the place and date. There was no accompanying motor, sensory, or visual impairment, clouding of consciousness, speech deficit, behavioural impairment, or convulsion. At $900 \mathrm{am}$, her general practitioner visited her at home. He noted memory impairment and temporal disorientation; blood pressure was $150 / 90 \mathrm{~mm} \mathrm{Hg}$. It was noted that the patient did not remember her sister's move to another nearby city 13 years before. During the remainder of the day, repetitive queries were noted by both her husband and her sister as well as by the hospital staff. Although no precipitating event was evident, the patient had felt tired for several days before the episode, and two potentially emotional circumstances existed: the prospect of being awarded a poetry prize the following day, and the unexpected death of her favourite poet and singer the day before.

At 12 noon, the patient was seen at the emergency ward of the university hospital of Caen. Language, behaviour, and somatic neurological examination were unremarkable. Unenhanced emergency CT of the head was normal, as were also the standard EEG and the ECG. At $400 \mathrm{pm}$, a neurologist noted an anterograde amnesia with temporal disorientation as well as a retrograde amnesia: the patient could not recall any of her personal events of the day, or of the day before (shopping in Bayeux, a walk at Omaha Beach). The patient had also forgotten about both the European Assembly elections (on 12 June 1994) and the just finished and locally vastly publicised 50th celebration of D Day (6 June 1994).

She was then sent for PET, which started at $630 \mathrm{pm}$ and was completed by $800 \mathrm{pm}$ (fig 1). Neuropsychological examination was done just

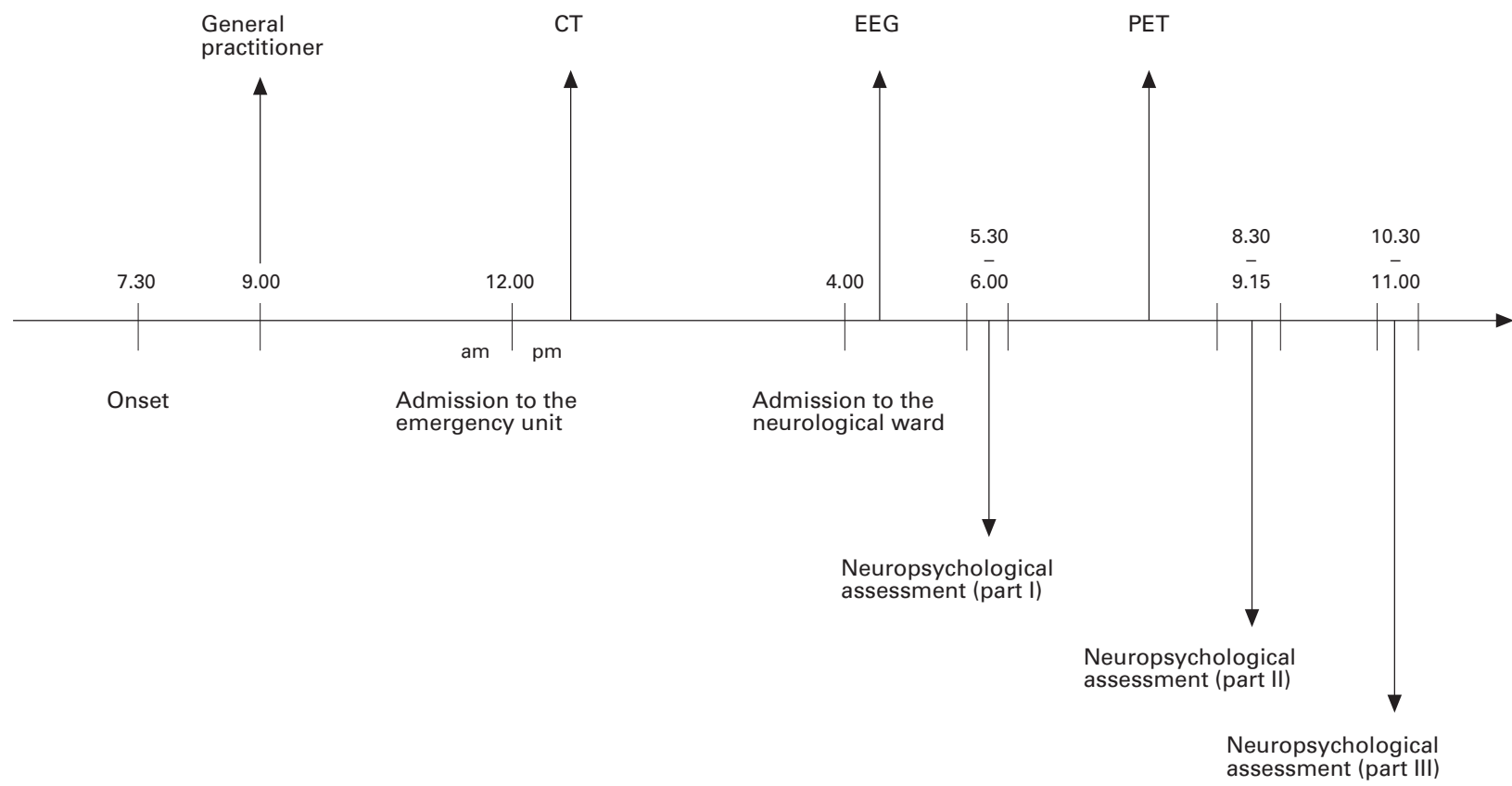

Figure 1 Time course of the different events related to the transient global amnesia episode. The neuropsychological assessment was performed in three parts: part I: explicit memory, word stem completion, general battery; part II: mirror reading (first session); part III: mirror reading (second session). 
Table 1 Performances of patient and matched control subjects (mean (SD)) in cognitive non-mnesic functions and explicit memory tests

\begin{tabular}{|c|c|c|c|}
\hline & \multirow[b]{2}{*}{ Controls } & \multicolumn{2}{|l|}{ Patient } \\
\hline & & Raw score & $z$ Score \\
\hline \multicolumn{4}{|l|}{ General cognitive functions: } \\
\hline Mental control $\ddagger$ & $12 \quad(0)$ & 12 & 0 \\
\hline Copy of Signoret's complex geometrical figure† & $11.9(0.24)$ & 12 & +0.42 \\
\hline Copy of geometrical figures $\ddagger$ & $10.73(1.14)$ & 10 & +0.64 \\
\hline Orientation $\ddagger$ & $11.5(0.86)$ & 12 & +0.58 \\
\hline Problem solving and semantic similarities $\ddagger$ & $11.27(0.87)$ & 12 & +0.84 \\
\hline \multicolumn{4}{|l|}{ Semantic memory: } \\
\hline Categorical verbal fluency $\ddagger$ & $11.53(0.86)$ & 8 & $-4.10^{\star \star}$ \\
\hline Naming $\ddagger$ & $10.9(0.96)$ & 12 & +1.14 \\
\hline \multicolumn{4}{|l|}{ Visual episodic memory: } \\
\hline $\begin{array}{l}\text { Six verbalisable line drawings } \ddagger \text { (free recall and } \\
\text { recognition) }\end{array}$ & $10.93(1.01)$ & 8 & $-2.90^{\star}$ \\
\hline Recall of complex geometrical figure & $8.57(1.24)$ & 3 & $-4.49^{\star \star}$ \\
\hline \multicolumn{4}{|l|}{ Verbal episodic memory: } \\
\hline Story recall $\dagger$ & $8.48(1.09)$ & 4.5 & $-3.65^{\star}$ \\
\hline \multicolumn{4}{|l|}{ Word learning } \\
\hline global score & $8.31(1.25)$ & 4.5 & $-3.05^{\star}$ \\
\hline Primacy effect $\$$ & $20.57(5.09)$ & 6 & $-2.86^{\star}$ \\
\hline \multicolumn{4}{|l|}{ Short term memory: } \\
\hline Word learning recency effect $\$$ & $3.71(2.87)$ & 6 & +0.80 \\
\hline Forward verbal span $\S$ & $5.1(1.07)$ & 7 & +1.77 \\
\hline
\end{tabular}

${ }^{\star} \mathrm{P}<0.01 ;{ }^{\star \star} \mathrm{P}<0.001$ reduced performance $v$ controls (see methods for calculations).

† From Signoret's "Batterie d'efficience cognitive". ${ }^{32}$

‡ From Signoret's "Batterie d'efficience mnésique" ${ }^{33}$

$\$$ Normative data from control sample of seven subjects (see methods for details).

The maximal possible score for all tests was 12 , except for the primacy and recency effects, and for the forward verbal span.

before and after PET scanning, while the patient was fully alert, and confirmed the existence of a major impairment of episodic memory (see next section). Thus the PET study was completed while the patient still exhibited severe anterograde amnesia.

At $1100 \mathrm{pm}$, after completion of the neuropsychological protocol, the patient still exhibited both anterograde and retrograde amnesia, although she clearly had entered the recovery phase. For instance, she still could not recall the death of her favourite poet-singer the day before. By the next morning, she was clinically normal. Four formal follow up neuropsychological examinations were performed, one and 14 days and four and 13 months after the episode (see below).

A three dimensional MRI, performed on 12 July, was normal, including both a detailed assessment of the hippocampus (with specially oriented cuts) and T2 weighted imaging. A cervical Doppler ultrasound investigation was also negative. A neurological examination at six months follow up was unremarkable. As of August 1996, her health has been unremarkable. She has a memory blank (amnesic gap) of the entire day.

NEUROPSYCHOLOGICAL EXAMINATION DURING THE ACUTE STATE

General assessment (table 1$)^{32} 33$

Mental control, copy of geometric figures, orientation, problem solving, semantic similarities, naming, and forward verbal span were not significantly altered (all scores were above the respective control means). Conversely, verbal fluency was significantly impaired (reduced output of correct items without errors nor perseverations), as were the verbal and the visual components of episodic memory. Regarding the word learning task, in addition to a significantly low global score, she exhibited an
Table 2 Mirror reading: results in controls (mean (SD)) and in the patient

\begin{tabular}{llll}
\hline & \multicolumn{3}{l}{ Patient } \\
\cline { 3 - 4 } & Controls $(n=7)$ & Performance & $z$ Score \\
\hline Slope 1 & $-0.028(0.012)^{\star}$ & -0.034 & $+0.5(\mathrm{NS})$ \\
Slope 2 & $-0.001(0.014)$ & -0.017 & $+1.14(\mathrm{NS})$ \\
Retention & $-0.185(0.082)^{\star}$ & -0.181 & $-0.05(\mathrm{NS})$ \\
\hline
\end{tabular}

$\star \mathrm{P}<0.001$ different from zero by paired $t$ test. Presentation is otherwise similar to table 1 .

impaired primacy effect but a preserved recency effect. Furthermore, the order of recall, which reflects strategy, was totally inconsistent across trials (data not shown). However, perseverations or overt frontal signs were not found.

Skill learning task (fig 2 and table 2)

Figure 2 and table 2 show the learning curves and corresponding quantitative values (slope 1 , slope 2, and retention index) respectively. Figure 2 shows that the control subjects learned the procedure rapidly during the first session, and showed no further learning at the second session. Our patient's data were not different and showed a better performance after learning relative to the control mean. In controls, the mean slope (on log transformed data) for the first session was significantly different from zero, indicating a significant learning effect (table 2). The corresponding slope for the patient was not significantly different from that of controls. For the second session, the slope in controls was not different from zero and showed a pronounced intersubject variability. The corresponding slope in the patient fell well within the normal range. The retention index was significantly different from zero in controls (indicating significant retention of the procedure from the first to the second session), and showed no alteration in the patient.

Word stem completion (table 3$)^{4647}$

The patients's performances in this task did not fall outside the $95 \%$ normal confidence limits for any score, and especially for priming effects.

FOLLOW UP NEUROPSYCHOLOGICAL ASSESSMENT Follow up neuropsychological assessments purposely focused on the functions which were altered during the episode, employing more formal tests whenever relevant. The morning after the episode, an IQ of 110 was obtained on Raven's progressive matrices ${ }^{48}$ and an MQ of 107 on the Wechsler memory scale (with normal scores in all the subtests). Word fluency, assessed with the same task as in the acute stage protocol, had normalised (13 and 22 names of animals in one and two minutes respectively). The performance in the copy of Rey's complex figure was normal (34/36), but its delayed recall after three minutes was still poor $(7 / 36, z=-2.5, \mathrm{P}<0.05)$.

Two weeks after the episode, the global mnesic score assessed with the BEM $144^{33}$ was strictly normal at 100.5, and verbal fluency was again normal. Four months after the episode, the patient was still clinically normal, the MQ was 101 and all subtests scores were normal, as 


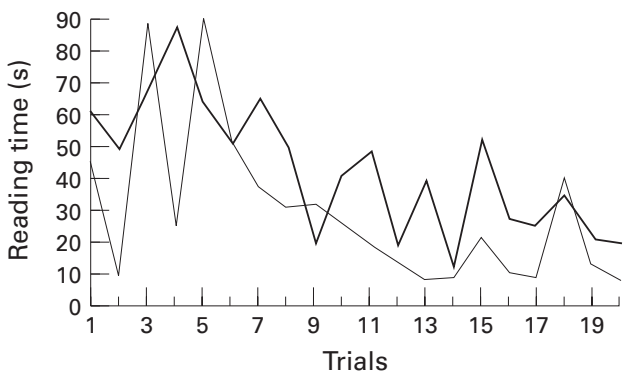

First session

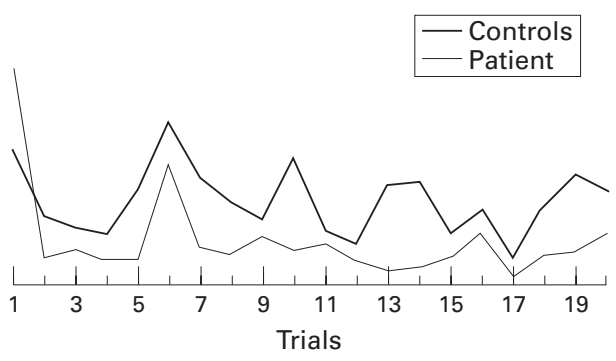

Second session

Figure 2 Mirror reading paradigm: learning curves for patient and control subjects (mean, $n=7)$; values shown are times spent to read each pair of words for 20 consecutive trials on each of two sessions (separated by a 90 minute interval). See text for quantitative results.

Table 3 Word stem completion: results for control subjects (mean (SD)) and patient

\begin{tabular}{llll}
\hline & & \multicolumn{2}{l}{ Patient } \\
\cline { 3 - 4 } & $\begin{array}{l}\text { Controls }\left(n=10^{\star}\right) \\
\text { age:61 (3.6) }\end{array}$ & Raw score & $z$ Score \\
\hline Primed words & $4.7(1.8)$ & 2 & -1.5 (NS) \\
Non-primed words & $1 \quad(0.8)$ & 0 & -1.2 (NS) \\
Priming effect & $3.7(1.7)$ & 2 & $-1 \quad(\mathrm{NS})$ \\
\hline
\end{tabular}

* Normative data from Desgranges et al. ${ }^{4647}$

Presentation is otherwise similar to table 1. Each maximum possible score is 10 .

was verbal fluency; however, performance in the delayed recall of Rey's figure was unchanged (6/36). With the BEC, there was normal performance in both free recall $(6 / 6)$ and recognition (6/6) of verbalisable line drawings, tests which were both significantly impaired in the acute stage (table 1). Thirteen months after the transient global amnesia episode, the neuropsychological examination was normal, including recall and recognition of the simple, non-verbalisable line drawings of the BEM, except for the delayed recall of Rey's and Signoret's complex geometric figures. Her poor performance in the recall of complex figures would reflect inadequate visuospatial abilities before the transient global amnesia episode, which the patient herself rated so since childhood. Furthermore, this peculiarity concerned preferentially the long term memory for complex geometric figures, but spared the delayed free recall of both verbalisable and simple non-verbalisable line drawings. ${ }^{49} 50$

PET STUDY

Appreciable head movements did not occur during the entire PET session; neither were movement artefacts evident on PET OEF images. Figure 3 shows the set of PET images with coregistered MRI. Visual interpretation suggested a reduction in both the $\mathrm{CMRO}_{2}$ and OEF over the left, compared with the right cortical convexity. The $\mathrm{CMRO}_{2}$ appeared especially reduced in the left frontal and temporal regions, as well as over the left lenticular nucleus. For CBF, there appeared a moderate reduction in the left, compared with the right lateral and posterior occipital cortex. The hippocampal area appeared unremarkable.

These findings from visual interpretation was confirmed by the objective ROI analysis; table 4 shows the main results. Although globally in the low range, the $\mathrm{CMRO}_{2}$ was significantly reduced relative to age matched controls only in the left lateral frontal cortex; no regional value for $\mathrm{CBF}$ or OEF fell outside the confidence limits from control data. The $\mathrm{CMRO}_{2}$ for the whole left neocortex was reduced relative to the right, the $\mathrm{L} / \mathrm{R} \%$ asymmetry falling just short of significance (right $=1.95 \mathrm{ml} / 100 \mathrm{~g} / \mathrm{min}$; left $=1.79 \mathrm{ml} / 100$ $\mathrm{g} / \mathrm{min} ; \mathrm{L} / \mathrm{R}=0.915, \mathrm{P}=0.06$ ). Regionally, the $\mathrm{CMRO}_{2}$ was significantly reduced in the left compared with the right lateral frontal cortex, lateral temporal cortex, and lentiform nucleus, with a similar although non-significant trend for the thalamus. An analysis of the individual cortical gyri to assess in further detail the distribution of metabolic abnormalities disclosed significant $\mathrm{CMRO}_{2}$ reductions in the left, compared with the right, middle, and inferior lateral frontal gyri and inferior lateral temporal cortex (data not shown). Relative to the right side, the OEF was reduced in the left lateral frontal cortex and lentiform nucleus $(\mathrm{P}<0.05$ for both), as well as in the left lateral temporal and lateral parietal cortex $(\mathrm{P}=0.06$

Table 4 PET results

\begin{tabular}{|c|c|c|c|c|c|c|c|c|c|}
\hline & \multicolumn{3}{|c|}{$C B F(\mathrm{ml} / 100 \mathrm{~g} \mathrm{~min})$} & \multicolumn{3}{|c|}{$\mathrm{CMRO}_{2}(\mathrm{ml} / 100 \mathrm{~g} \mathrm{~min})$} & \multicolumn{3}{|l|}{$O E F$} \\
\hline & Right & Left & $L / R \%$ & Right & Left & $L / R \%$ & Right & Left & $L / R \%$ \\
\hline Lateral frontal cortex & 29 & 28 & -3 & 1.93 & $1.77^{\star}$ & $-8^{\star \star}$ & 0.41 & 0.39 & $-6^{\star}$ \\
\hline Lateral temporal cortex & 29 & 28 & -3 & 2.08 & 1.84 & $-12^{\star}$ & 0.45 & 0.41 & $-9 \dagger$ \\
\hline Lateral parietal cortex & 24 & 26 & $+8^{\star}$ & 1.72 & 1.64 & -5 & 0.44 & 0.40 & $-9+$ \\
\hline Occipital cortex $\ddagger$ & 31 & 26 & $-16^{\star}$ & 2.08 & 1.94 & -7 & 0.40 & 0.45 & $+13^{\star}$ \\
\hline Hippocampus & 29 & 28 & -3 & 1.70 & 1.59 & -6 & 0.36 & 0.35 & -3 \\
\hline Thalamus & 37 & 30 & -19 & 2.08 & 1.65 & -21 & 0.34 & 0.34 & 0 \\
\hline Lentiform nucleus & 36 & 29 & $-19^{\star}$ & 2.25 & 1.50 & $-33^{\star \star}$ & 0.39 & 0.32 & $-18^{\star}$ \\
\hline
\end{tabular}

${ }^{\star} \mathrm{p}<0.05,{ }^{\star \star} \mathrm{p}<0.01$ with respect to confidence intervals calculated in 10 age matched normal controls (see methods) $+\mathrm{p}=0.06$; $\ddagger$ this region includes the lateral occipital cortex and the cuneus. $\mathrm{CBF}=$ cerebral blood flow; $\mathrm{CMRO}_{2}=$ cerebral metabolic rate of oxygen; OEF $=$ oxygen extraction fraction. 

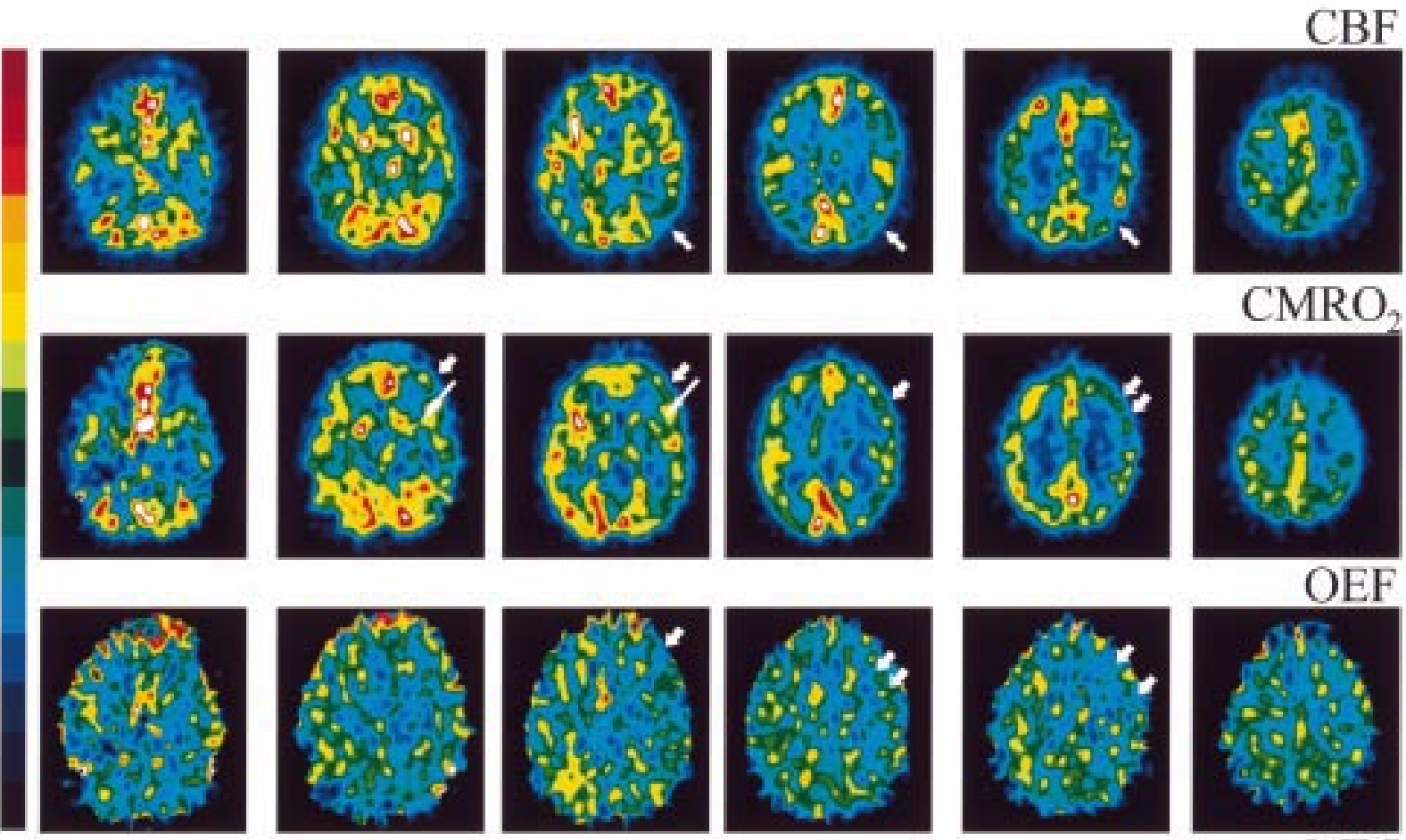

OEF

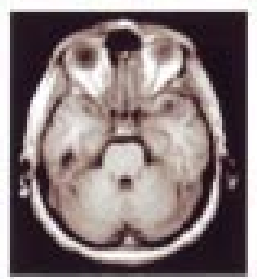

GI - 4 mm

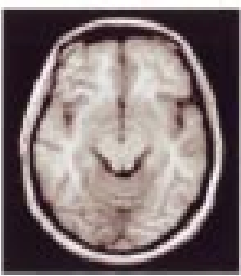

$\mathrm{GI}+8 \mathrm{~mm}$

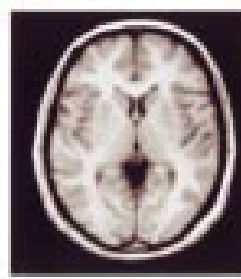

$\mathrm{GI}+20 \mathrm{~mm}$

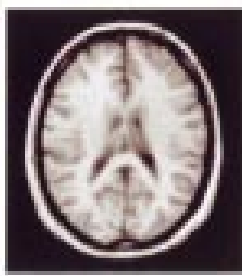

GI + $32 \mathrm{~mm}$

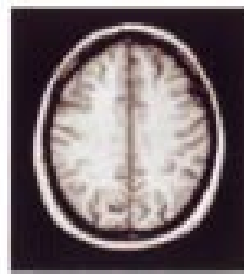

GI + 44 mm

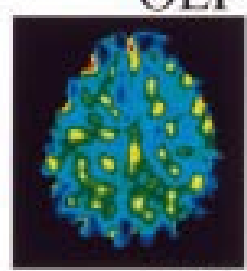

MRI

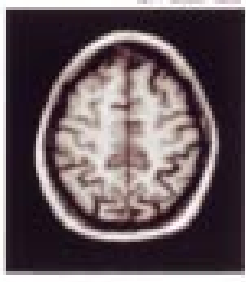

$\mathrm{GI}+56 \mathrm{~mm}$

Figure 3 Axial PET images (upper three rows) of cerebral blood flow (CBF), oxygen consumption (CMRO ${ }_{2}$ ), and oxygen extraction fraction (OEF), and coregistered T1 weighted MRI (bottom row). The PET images are parametric maps displayed in a pseudocolour scale shown on the right, ranging from black for zero to white for maximal pixel value (range indicated for each variable; $C B F$ and $C M R O_{2}$ are in ml/100 g/min and OEF is dimensionless). All images were obtained parallel to the Glabella-Inion line and at midscan distances shown below the MRI cuts. Visual inspection of the PET images suggests reduced CBF in the left lateral occipitotemporal cortex (arrows) reduced CMRO, in the left frontal and temporal-occipital cortex (small arrows) as well as over the left lentiform nucleus (long arrows), and reduced OEF in the left frontal area. The hippocampal area appears unremarkable. These findings were largely confirmed by the quantitative analysis (table 4), which, however, showed additional significant changes. The MRI performed four weeks after the PET was normal.

for both), whereas the CBF was significantly reduced in the left lateral occipital cortex. For the hippocampus, there was not even a trend for changes in any of the variables assessed (table 4).

Over and above these findings, which essentially confirmed the visual interpretation, the ROI analysis disclosed the following additional significant changes (all $\mathrm{P}<0.05)$ : (1) a significant increase in the OEF in the left occipital cortex, (2) a significant increase in CBF in the left parietal cortex, and (3) a significant decrease in $\mathrm{CBF}$ in the left lentiform nucleus. Not shown in table 4, there was a lack of significant changes in the caudate nucleus and the cerebellum.

\section{Discussion}

This is the first study to report on a patient with transient global amnesia fulfilling the operational criteria for transient global amnesia of Hodges and Warlow, ${ }^{21}$ examined in the acute stage with a prospective protocol comprising both a brief neuropsychological assessment (including tests of skill learning and priming effects) and PET. Nausea and headache occur at times during transient global amnesia. ${ }^{251}$ No epileptic feature was noted at any time and there was no history of head injury. All medical investigations, including EEG, CT, and detailed MRI, were strictly normal, and no further episode has occurred with a follow up over two years.

NEUROPSYCHOLOGICAL DATA

The patient's neuropsychological assessment confirmed the presence of severe anterograde amnesia and showed a significant impairment of episodic LTM (for both verbal and visual components), whereas STM was strictly intact. The preserved recency effect but altered primacy effect, showing a clear cut dissociation between LTM and STM, is consistent with most previous transient global amnesia case reports (see introduction). The patient's categorical fluency was also significantly reduced 
during the episode but had normalised as early as the morning after. Impaired verbal fluency (either categorical or formal) has been previously reported during ${ }^{517}$ or immediatly after ${ }^{13}$ a transient global amnesia episode, although this certainly is not a consistent finding. ${ }^{341011}$ Categorical fluency deficits can result from a perturbation of either semantic memory or retrieval strategy. The preservation of all other language functions in our patient (namely, spontaneous verbal expression and comprehension, confrontation naming, and semantic similarities) favours the integrity of semantic stores as also supported by the normality of lexical-semantic priming effects. This interpretation would also fit well with Hodges' ${ }^{3}$ finding of intact semantic memory in his two patients. Thus although we cannot formally exclude the existence of a mild semantic impairment, the reduction of verbal fluency in our patient most likely reflected an alteration in strategy, which would account for the observed inconsistency in recall order across trials in the word learning test.

In our study, we used two distinct paradigms to assess skill learning and priming effects. Both these components of implicit memory were preserved. Using identification of fragmented digits, Goldenberg et $a l^{14}$ were the first to claim preservation of perceptual skill learning in transient global amnesia. Kazui et $a l^{18}$ reported preservation of cognitive, perceptualmotor, and perceptual-verbal procedural memory, using the Tower of Toronto, a drawing skill, and reading skill tests respectively; however, the last did not involve the aquisition of a new skill and therefore would not be rated by most as a procedural memory test. Thus the present study seems to be the first to have formally assessed perceptualverbal skill learning during transient global amnesia; we used the mirror-reading paradigm, which is well recognised to assess this process. Both the acquisition and the retention of this skill were similar to control subjects (see fig 2; note that the better performance of control subjects in the first trial of session II may reflect an involvement of declarative memory, ${ }^{52}$ which was impaired in the patient).

For priming effects, we used an original version of the word stem completion paradigm, specifically designed to investigate lexicalsemantic priming effects, which did not fall outside the (admittedly wide ${ }^{53}$ ) normal range. Recently, both Kazui et $a l^{18}$ and Kapur et $a l^{19}$ showed preservation of perceptual priming in three and one cases, respectively. Overall, therefore, we document for the first time the preservation of both perceptual-verbal skill learning and lexical-semantic priming effects in transient global amnesia.

PET FINDINGS

A PET study was carried out while the patient was still severely amnesic, as documented by the cognitive tests performed during and after scanning. All the changes were confined to the left cerebral hemisphere, although with different patterns of CBF-CMRO ${ }_{2}$ uncoupling from region to region. Specifically, there was a meta- bolic depression maximum in the left frontal cortex (especially affecting the middle and inferior frontal gyri), left temporal cortex (especially inferior), and left lentiform nucleus. In these regions, the CBF was fully preserved in the former two and reduced, (but less so than the $\mathrm{CMRO}_{2}$ ) in the third region respectively, with consequently a reduction in the OEF in all three regions. Other abnormalities included (1) a mildly increased CBF with reduced OEF but normal $\mathrm{CMRO}_{2}$ in the left parietal cortex, and (2) a reduction in CBF with increased $\mathrm{OEF}$, again with normal $\mathrm{CMRO}_{2}$, in the left occipital cortex. There was a non-significant trend for reduced $\mathrm{CBF}$ and $\mathrm{CMRO}_{2}$ in the left thalamus. Finally, the hippocampal area exhibited not even a trend for changes in any variable; this does not reflect a lack of sensitivity of our methodology to changes in the hippocampal region, as shown previously by, for example, age related reductions in hippocampal $\mathrm{CMRO}_{2}$ correlated with verbal episodic memory declines. ${ }^{385}$

This report of predominantly neocortical changes with unaffected hippocampal physiology during transient global amnesia, although at first sight surprising, is not isolated. As stated in the introduction, several previous functional neuroimaging studies, including our previous PET report, ${ }^{23}$ have reported neocortical hypoperfusion/hypometabolism, in some instances associated with ipsilateral or bilateral thalamic changes, yet without hippocampal changes. Although the last may have occurred at earlier times into the episode, the patient was severely amnesic during PET scanning. Thus the present report further strengthens the idea that, at least in some patients, the clinical expression of a global but transient amnesia may correspond to predominantly neocortical dysfunction; this distribution of physiological changes in transient global amnesia is at variance with the topography of brain damage in the PAS, despite a very similar neuropsychological pattern. This discrepancy may in part reflect the single major difference between transient global amnesia and PAS-namely, that the latter usually represents the final, stable phase of an initially more diffuse and complex neuropsychological picture; in other words, the clinical expression of a given acute focal brain dysfunction may considerably overstate that which permanently endures once brain reorganisation has taken place.

Compared with the right side, the relative preservation (or even increase) of CBF with mildly but significantly decreased OEF in the left frontal, temporal, and parietal cortices would suggest a flow metabolism uncoupling compatible with, for example, prior ischaemia, prior seizure, or prior spreading depression. ${ }^{55-57}$ Previously, Volpe et $a l^{24}$ also reported a reduced $\mathrm{OEF}$ in the hippocampal region of their patient with transient global amnesia; however, in the case of Baron et $a l^{23}$ the OEF was normal. Thus different pathophysiological mechanisms may trigger transient global amnesia, or, alternatively, each patient is "snapshot" with PET at different phases of one (and the same) process. The presence of mild but significant 
changes with reduced $\mathrm{CBF}$ and increased $\mathrm{OEF}$ in the left compared with the right occipital cortex is of great interest as it suggests the mechanism underlying transient global amnesia in this case also included oligemia ${ }^{5657}$ as one of its expressions. The aura phase of classic migraine is characterised by a narrow wave of hyperperfusion slowly spreading from the occipital cortex towards the forebrain and leaving in its aftermath a prolonged and extensive hypoperfusion ${ }^{58}{ }^{59}$ with (in the single instance of protracted aura captured by PET) focally increased OEF.$^{6061}$ These changes have been largely interpreted as reflecting the human equivalent of the phenomenon of spreading depression of Leão. ${ }^{62}$ The nature and complex pattern of associated abnormalities in CBF, $\mathrm{OEF}$, and $\mathrm{CMRO}_{2}$ in our patient would be compatible with these migrainous phenomena, and spreading depression has been speculated previously as one trigger of transient global amnesia. ${ }^{63}$ From a clinical point of view, there are many arguments which point towards a link between transient global amnesia and migraine, both epidemiological and symptomwise $^{264}$ and our present findings would add a pathophysiological argument as one further support to this hypothesis.

The present findings also clearly illustrate that in the acute phase of transient global amnesia the CBF may be uncoupled from $\mathrm{CMRO}_{2}$ (in either direction). Thus perfusion imaging studies without concomittant assessment of metabolism (as with SPECT) may mislead the interpretation in terms of synaptic function.

\section{NEUROPSYCHOLOGY-METABOLISM RELATIONS} Impaired episodic memory

Although both clinical and animal neuropsychology have shown the implication of the hippocampal complex in episodic memory, ${ }^{65}$ the $\mathrm{CMRO}_{2}$ in the hippocampal region was not significantly altered in our patient, despite concurrent massive episodic amnesia. As stated above, this finding is, however, consistent with several reports, ${ }^{23}$ although hippocampal changes were effectively detected in others. ${ }^{218}$ Furthermore, as PET or SPECT in other conditions (for example, normal aging, PAS, Alzheimer's disease) have documented significant relations between hippocampal area metabolism and episodic memory deficits, ${ }^{546667}$ the imaging technology is not to be called into question. PET activation studies in normal subjects did not systematically show involvement of the hippocampus in episodic memory tasks. ${ }^{689}$ The implication of the hippocampus in episodic memory is probably much more complex than previously thought and may not be systematically required. ${ }^{70}$ It is worth recalling in this context that a typical PAS may develop despite an intact hippocampus if the lesion affects other parts of the episodic memory (limbic) network-for example, the diencephalic area. ${ }^{71}$ In the present patient, there was a trend for left thalamic hypometabolism which however did not reach significance. In the case reported by Baron et al, ${ }^{23}$ right sided tandem prefrontal thalamic hypometabolism was found.

It is tempting, even if speculative, to relate the deficit in episodic memory in our patient with hypometabolism in the left prefrontal cortex. Thus although subjects with frontal lobe impairment do not exhibit genuine PAS, they do have difficulties in making temporal order judgements (frequency and recency assessment), increased susceptibility to interference, ${ }^{72}$ impaired free recall, but preserved recognition ${ }^{73}$ and "source" amnesia. ${ }^{74}$ Furthermore, PET activation studies have recently clarified the role of the prefrontal cortex in memory. Thus according to the hemispheric encoding retrieval asymmetry (HERA) model, ${ }^{75}$ the left and right prefrontal cortices are involved in the operations of encoding and retrieval of episodic information respectively. It is of note that the hypometabolic zone in the left prefrontal cortex in our patient affected the inferior frontal gyrus, precisely where Shallice $e t a l^{68}$ found a significant activation during episodic memory encoding in normal subjects. Our PET results would thus be consistent with an encoding deficit; however, we did not specifically evaluate encoding versus retrieval of new episodic information. In the single detailed PET report on transient global amnesia published to date, ${ }^{23}$ a right prefrontal hypometabolism was found; unfortunately, a neuropsychological assessment could not be concurrently performed. SPECT reports have occasionally mentioned either right or left prefrontal hypoperfusion during transient global amnesia, ${ }^{2829}$ but encoding versus retrieval was not assessed. Thus the hypothesis of a causal relation between prefrontal hypometabolism and episodic memory impairment during transient global amnesia will have to be tested in future work. However, it is unclear whether the left prefrontal hypometabolism in our patient also accounts for the presence of retrograde amnesia, which must reflect a retrieval impairment; however, this retrieval mechanism may well differ from the one operative in episodic tasks assessing anterograde amnesia, as suggested by dissociations in cases of pure retrograde amnesia. $^{76}$

\section{IMPAIRED VERBAL FLUENCY}

During the episode, our patient exhibited an impaired categorical verbal fluency. This was the only significant cognitive impairment apart from the deficit of episodic memory. It is likely that this impairment was related to the left prefrontal or temporal hypometabolism disclosed by PET. Previously, Goldenberg ${ }^{2}$ had reported a patient with impaired verbal fluency together with left frontal hypoperfusion during a transient global amnesia episode. Aphasic patients with left temporal damage exhibit anomia, and several PET activation studies have localised semantic representations for words and objects in the left temporal lobe. ${ }^{7778}$ However, as discussed above, the neuropsychological data were more consistent with an impairment in verbal fluency from strategy rather than semantic knowledge alteration, which would point towards the left frontal lobe. Accordingly, left prefrontal cortex activations 
have been consistently found across various PET paradigms involving either categorical verbal fluency ${ }^{79}$ or a word generation paradigm. ${ }^{80-82}$ It is thought that the left cortex is specifically engaged in the search, manipulation, and mental organisation of semantic information, either automatic or willed, including retrieval from semantic stores. ${ }^{83}$ This function of the left dorsolateral prefrontal cortex is incorporated in the HERA model (elaborated by Tulving $e t a l^{75}$ and supported by Shallice $e t$ $\left.a l^{68}\right)$.

INTACT PERCEPTUAL-VERBAL SKILL LEARNING The pattern of metabolic depression observed in the patient, sparing the cerebellum and the caudate, would be compatible with preserved perceptual-verbal skill learning. Thus neuropsychological studies suggest a contribution of the cerebellum ${ }^{84}$ and of a corticostriatal system $^{8586}$ in skill learning. Furthermore, caudate nucleus lesions impair the acquisition of mirror reading but not target pursuit skills, and putaminal lesions the reverse pattern. ${ }^{87}$ PET activation studies in healthy subjects have attributed a major role to the cortical-striatalcerebellar motor system in motor skill learning. ${ }^{88}$ To our knowledge, however, no activation study has so far specifically focused on perceptual-verbal skill learning.

PRESERVED LEXICAL-SEMANTIC PRIMING EFFECTS Because a selective impairment in priming effects as a result of brain damage seems at best very rare, the neural substrates of this intriguing phenomenon remain largely unknown to date. However, the fact that priming effects are intact in PAS indicates that the limbic system (hippocampus, diencephalon, anterior cingulate cortex) is not required for this function. Lexical-semantic priming is impaired in Alzheimer's disease ${ }^{89} 90$ suggesting that widespread lesions are required in association with other cognitive deficits. Our patient exhibited left temporal cortex hypometabolism despite unaltered lexical-semantic priming effects. It may be that the temporal metabolic depression was too mild, or too circumscribed, or both, to result in impaired lexical-semantic priming.

We acknowledge the help from the Cyclotron staff, PET camera technicians, and radiographers of Cyceron, as well as Mr D Luet and Mrs B Aubry for help with preparation of the manuscript and the staff of the emergency unit of the University Hospital of and the

1 Hodges JR, Warlow CP. The aetiology of transient global amnesia: a case-control study of 114 cases with prospective amnesia: a case-control study of 114

2 Goldenberg G. Transient global amnesia. In: Baddeley AD, Wilson BA, Watts FN, eds. Handbook of memory disorders. Chichester: J Wiley, 1995:109-33.

3 Hodges JR. Semantic memory and frontal executive function during transient global amnesia. $\mathcal{f}$ Neurol Neurosurg Psychiatry 1994;57:605-8.

4 Wilson RS, Koller W, Kelly MP. The amnesia of transient global amnesia. fournal of Clinical Neuropsychology, 1980;9 (suppl): 19-20.

5 Caffara P, Moretti G, Mazzucchi A, Parma M. Neuropsychological testing during a transient global amnesia episode and its follow-up. Acta Neurol Scand 1981;63:44-50.

6 Regard M, Landis T. Transient global amnesia: neuropsychological dysfunction during attack and recovery in two "pure" cases. I Neurol Neurosurg Psychiatry 1984;47:66872 .

7 Gallassi R, Lorusso S, Stracciari A. Neuropsychological findings during a transient global amnesia attack and its follow-up. Ital f Neurol Sci 1986;7:45-9.
8 Stracciari A, Rebucci GG, Gallassi R. Transient global amnesia: neuropsychological study of a "pure” case. $\mathscr{f} \mathrm{Neu}$ rol 1987;234:126-7.

9 Kritchevsky M, Squire LR, Zouzounis JA. Transient global amnesia: characterization of anterograde and retrograde amnesia. Neurology 1988;38:213-9.

10 Meador KJ, Loring DW, King DW, Nichols FT. The P3 evoked potential and transient global amnesia. Arch Neurol 1988;45:465-7.

11 Hodges JR, Ward CD. Observations during transient global amnesia: a behavioural and neuropsychological study of five cases. Brain 1989;112:595-620.

12 Kritchevsky M, Squire LR. Transient global amnesia: evidence for extensive, temporally graded retrograde amnesia. Neurology 1989;39:218-31.

13 Stilhard G, Landis T, Schiess R, Regard M, Sialer G. Bitemporal hypoperfusion in transient global amnesia: $99 \mathrm{~m}$-tcHMPAO SPECT and neuropsychological findings during and after an attack. $\mathcal{F}$ Neurol Neurosurg Psychiatry 1990;53: and after ar $339-42$.

14 Goldenberg G, Podreka I, Pfaffelmeyer N, Wessely P, Deecke L. Transient ischemia in transient global amnesia: a SPECT study. Neurology 1991;41:1748-52.

15 Tanabe H, Hashikawa K, Nakngawa Y, Ikeda M, Yamamoto $\mathrm{H}$, Harada $\mathrm{K}$, et al. Memory loss due to transient hypoperfusion in the medial temporal lobes including hippocampus. Acta Neurol Scand 1991;84:22-7.

16 Evans J, Wilson B, Wraight EP, Hodges JR. Neuropsychological and SPECT scan findings during and after transient global amnesia: evidence for the differential impairment of remote episodic memory. F Neurol Neurosurg Psychiatry 1993;56:1227-30

17 Lin KN, Liu RS, Yeh TP, Wang SJ, Liu HC. Posterior ischemia during an attack of transient global amnesia. 3;24:1093-5.

18 Kazui H, Tanabe H, Ikeda Y, Nakagawa Y, Shiraishi J, Hashikawa K. Memory and cerebral blood flow in cases of transient global amnesia during and after the attack. Behav Neurol 1995;8:93-101.

19 Kapur N, Abbott P, Footitt D, Millar J. Long-term perceptual priming in transient global amnesia. Brain Cogn 1996; 31:63-74

20 Byer JA, Crowley WJ. Musical performance during transient global amnesia. Neurology 1980;30:80-2.

21 Hodges JR, Warlow CP. Syndromes of transient global amnesia: towards a classification: a study of 153 cases. $\mathcal{F}$ Neurol Neurosurg Psychiatry 1990;53:834-43.

22 Tulving E, Schacter DL. Priming and human memory systems. Science 1990;247:301-5.

23 Baron JC, Petit-Taboué MC, Le Doze F, Desgranges B, Ravenel N, Marchal G. Right frontal cortex hypometabolism in transient global amnesia: a PET study. Brain 1994; 117:545-52.

24 Volpe BB, Herscowitch P, Raichle ME, Hirst W, Gazzaniga MS. Cerebral blood flow and metabolism in human amnesia. F Cereb Blood Flow Metab 1983;3(suppl 1):S5-6.

25 Heiss WF, Pawlik G, Holtoff V, Kessler J, Szelies B. PET correlates of normal and impaired memory functions. Cerebrovasc Brain Metab Rev 1992;4:1-27.

26 Matsuda H, Higashi S, Tsuji S, Sumiya H, Miyauchi T, Hisada K, Yamashita J. Hight resolution Tc-99m HMPAO SPECT in a patient with transient global amnesia. Clin Nucl Med 1993;18:46-9.

27 Sakashita Y, Sugimoto T, Taki S, Matsuda H. Abnormal cerebral blood flow following transient global amnesia. $\mathcal{f}$ Neurol Neurosurg Psychiatry 1993;56:1327.

28 Trillet M, Croisile B, Philippon B, Vial C, Laurent B. Guillot M. Ictus amnésique et debits sanguin cerebraux. Rev Neurol 1987;143:536-9.

29 Fayad P, Kain T, Hoffer P, Smith E, Brass LM. SPECT findings in transient global amnesia (TGA) [abstract]. Neurology 1990;40(suppl 1):171.

30 Baron JC. Positron emission tomography (PET) in acute ischemic stroke: pathophysiological and clinical implications. In: LR Caplan, ed. Brain ischemia: basic concepts and clinical relevance. Berlin: Springer-Verlag, 1995:2:19-27.

31 Caplan LR. Transient global amnesia. In: Fredericks JAM, ed. Handbook of clinical neurology. Amsterdam: Elsevier, 1985:205-18

32 Signoret JL, Allard M, Benoit N, Bonvarlet M, Eustache F. Evaluation des troubles de mémoire et des désordres cognitifs associés. BEC 96. Paris: Fondation IPSEN, 1989.

33 Signoret JL. Batterie d'efficience mnésique. BEM 144. Collection Esprit et Cerveau. Paris: Fondation IPSEN. Amsterdam: Elsevier, 1991.

34 Tulving E, Colotla VA. Free recall of trilingual lists. Cognitive Psychology 1970;1:86-98.

35 Cohen NJ, Squire LR. Preserved learning and retention of pattern analyzing skill in amnesia: dissociation of knowing how and knowing what. Science 1980;210:207-10.

36 Shimamura A, Salmon D, Squire L, Butters N. Memory dysfunction and word priming in dementia and amnesia. Behav Neurosci 1987;101:347-51.

37 Centre de Recherche pour un Trésor de la Langue Francaise. Etudes statistiques sur le vocabulaire français. Dictionnaire des fréquences: vocabulaire littéraire des XIXème et XXème siècles. Nancy: CNRS, 1971.

38 Marchal G, Rioux P, Petit-Tabouè MC, Sette G, Travére JM, Le Poec C, et al. Regional cerebral oxygen consumption, blood flow, and blood volume in healthy human aging. Arch Neurol 1992;49:1013-20.

39 Frackowiak RSJ, Lenzi GL, Jones T, Heather JD. Quantitative measurement of regional cerebral blood flow and metabolism in man using ${ }^{15} \mathrm{O}$ and positron emission 
tomography: theory, procedure, and normal values. $\mathcal{F} \mathrm{Com}$ put Assist Tomogr 1980;4:727-36.

40 Mazyer B, Treos A. Physical characteristics of TTVO3, a new high spatial resolution time-of-flight positron tomograph. IEEE Tran Nucl Sci 1990;37:778-82.

41 Fox PT, Perlmutter JS, Raichle ME. A stereotactic method of anatomical localization for positron emission tomography. $\mathcal{F}$ Comput Assist Tomogr 1985;9:141-53.

42 Woods RP, Cherry SR, Mazziotta JC. A rapid automated algorithm for accurately aligning and reslicing positron emission tomography images. F Comput Assist Tomogr 1992; 16:620-33.

43 Pantano P, Baron JC, Crouzel C, Collard P, Sirou P, Samson $\mathrm{Y}$. The ${ }^{15} \mathrm{O}$ continuous inhalation method: correction for intravascular signal using $\mathrm{C}^{15} \mathrm{O}$. Eur f Nucl Med 1985;10: 387-91.

44 Allain P, Traveré JM, Baron JC, Bloyet D. Accurate PET positioning with reference to MRI and neuroanatomical positioning with reference to MR eds. Ouantification of brain function. Tracer kinetics and image eds. Quantification of brain function. Tracer kinetics and image

45 Talairach J, Tournoux P. Co-planar stereotaxic atlas of the 1988.

46 Desgranges B, Eustache F, Rioux P, Effets de l'âge et du niveau d'étude sur differénts sous-systèmes mnésiques. L'Année Psychologique 1994;94:345-68.

47 Desgranges B, Eustache F, Rioux P, De La Sayette V, Lech evalier B. Memory disorders in Alzheimer's disease and the organization of human memory. Cortex 1996;32:387-412.

48 Raven J. Progressive matrices. Edition francaise, 1981. Issy-lesMoulineaux: Editions Scientifiques et Psychotechniques, 1983.

49 Hodges JR, Oxbury SM. Persistent memory impairment following transient global amnesia. $\mathcal{F}$ Clinical Exp Neuropsychol 1990;12:904-20.

50 Mazzucchi A, Moretti G, Caffara P, Parma M. Neuropsychological functions in the follow up of transient global chological functions in the follow

51 Markowitsch HJ. Transient global amnesia and related disorders. Stuttgart: Hogrefe and Huber, 1990.

52 Harrington DL, Haaland KY, Yeo RA, Marder E. Procedural memory in Parkinson's disease: impaired motor but not visuoperceptual learning. F Clinical Exp Neuropsychol 1990;12:323-39.

53 Deweer B, Ergis AM, Fossati P, Pillon B, Boller F, Agid Y Dubois B. Explicit memory, procedural learning and lexica priming in Alzheimer's disease. Cortex 1994;30:113-26.

54 Eustache F, Rioux P, Desgranges B, Marchal G, PetitTaboué MC, Dary M, et al. Healthy aging, memory subsystems and regional cerebral oxygen consumption. Neuropsychologia 1995;33:867-87.

55 Lassen NA. The luxury-perfusion syndrome and its possible relation to acute metabolic acidosis localised within the brain. Lancet 1966; i:1113-5.

56 Lassen NA. Pathophysiology of brain ischemia as it relates to the therapy of acute ischemic stroke. Clin Neuropharmacol 1990;13:S1-85.

57 Baron JC. Méthodes actuelles de mesure du débit sanguin et du métabolisme cérébral régional chez l'homme. Encyclopédie Médico-Chirurgicale (Neurologie) 1992;17-035A30:6.

58 Olesen J, Larsen B, Lauritzen M. Focal hyperemia followed by spreading oligemia and impaired activation of $\mathrm{rCBF}$ in classic migraine. Ann Neurol 1981;9:344-52.

59 Woods RP, Iacoboni M, Mazziotta JC. Bilateral spreading cerebral hypoperfusion during spontaneous migraine headache. N Engl f Med 1994;331:1689-92.

60 Herold S, Gibbs JM, Jones AKP, Brooks DJ, Frackowiak RSJ, Legg NJ. Oxygen metabolism in migraine. 7 Cereb Blood Flow Metab 1985;5:S445.

61 Friberg L, Olesen J, Lassen NA, Skyhoj T, Karle A. Cerebral oxygen extraction, oxygen consumption, and regional cerebral blood flow during the aura phase of migraine. Stroke 1994;25:974-9.

62 Lauritzen $M$. Pathophysiology of the migraine aura: the Lauritzen M. Pathophysiology of the migraine aura:

63 Olesen J, Jorgensen MB. Leao's spreading depression in the hippocampus explains transient global amnesia - a hypothhippocampus explains transient global am

64 Caplan LR, Chedru F, Lhermitte F, Mayman C. Transien global amnesia and migraine. Neurology 1981;31:1167-70.

65 Squire LR. Memory and the hippocampus: a synthesis from findings with rats, monkeys, and human. Psychol Rev 1992 99:195-231.
66 Perani D, Bressi S, Cappa SF, Vallar G, Alberoni M, Grassi $\mathrm{F}$, et al. Evidence of multiple memory systems in the human brain. A $\left[{ }^{18} \mathrm{~F}\right]$ FDG PET metabolic study. Brain 1993;116: 903-19.

67 Ohnishi T, Hoshi H, Nagamachi S, Jinnouchi S, Flores II LG, Futami S, Watanabe K. High-resolution SPECT to assess hippocampal perfusion in neuropsychiatric diseases. f Nucl Med 1995;36:1163-9.

68 Shallice T, Fletcher P, Frith CD, Grasby P, Frackowiak RSJ, Dolan RJ. Brain regions associated with acquisition and retrieval of verbal episodic memory. Nature 1994;368:6335.

69 Buckner RL, Petersen SE, Ojemann JG, Miezin FM, Squire LR, Raichle ME. Functional anatomical studies of explicit and implicit memory retrieval tasks. F Neurosci 1995;15:1229.

70 Fletcher PC, Frith CD, Grasby PM, Shallice T, Frackowiak RSJ, Dolan SJ. Brain systems for encoding and retrieval of auditory-verbal memory. An in vivo study in humans. Brain 1995;118:401-16.

71 Zola-Morgan S, Squire LR. Neuroanatomy of memory. Annu Rev Neurosci 1993;16:547-63.

72 Smith ML, Leonard G, Crane J, Milner B. The effects of frontal- or temporal- lobe lesions on susceptibility to interference in spatial memory. Neuropsychologia 1995;33:27585

73 Janowsky JS, Shimamura AP, Kritchevski M, Squire LR. Cognitive impairment following frontal lobe damage and its relevance to human amnesia. Behav Neurosci 1989;103: 548-60.

74 Janowsky JS, Shimamura AP, Squire LR. Source memory mpairment in patients with frontal lobe lesions. Neuropsychologia 1989;27:1043-56.

75 Tulving E, Kapur S, Craik FIM, Moscovitch M, Houle S. Hemispheric encoding/retrieval asymmetry in episodic memory: positron emission tomography findings. Proc Natl Acad Sci USA 1994;91:2016-20.

76 Kapur N. Focal retrograde amnesias in neurological disease: a critical review. Cortex 1993;29:217-34.

77 Damasio H, Grabowski TJ, Tranel D, Hichwa RD, Damasio AR. A neural basis for lexical retrieval. Nature 1996;380: $499-505$.

78 Martin A, Wiggs CL, Ungerleider LG, Haxby JV. Neural correlates of category-specific knowledge. Nature 1996; 379:649-52.

79 Frith CD, Friston K, Liddle PF, Frackowiak RSJ. A PET study of word finding Neuropsychologia 1991;29:1137-48.

80 Petersen SE, Fox PT, Posner MI, Mintun M, Raichle ME. Positron emission tomographic studies of the processing of single words. Fournal of Cognitive Neuroscience 1989;2:153-

81 Raichle ME, Fiez JA, Videen TO, MacLeod AMK, Pardo JV, Fox PT, Petersen SE. Practiced-related changes in human brain functional anatomy during nonmotor learning. Cereb Cortex 1994;4:8-26.

82 Klein D, Milner B, Zatorre RJ, Meyer E, Evans AC. The neural substrates underlying word generation: a bilingual functional imaging study. Proc Natl Acad Sci USA 1996;92: 2899-903.

83 Kapur S, Rose R, Liddle PF, Zipursky RB, Brown GM, Stuss D, Houle S, Tulving E. The role of the left prefrontal cortex in verbal processing: semantic processing or willed action? Neuroreport 1994;5:2193-6.

84 Daum I, Ackermann H, Schugens MM, Reimold C, Dichgans J, Birbaumer N. The cerebellum and cognitive functions in humans. Behav Neurosci 1993;107:411-9.

85 Heindel W, Butters N, Salmon D. Impaired learning of a motor skill in patients with Huntington's disease. Behav Neurosci 1988;102:141-7.

86 Salmon D, Butters N. Neurobiology of skill and habit learning. Curr Opin Neurobiol 1995;5:184-90.

87 Deweer $B$. Intérêt de la dissociation entre démences corticales et démences sous-corticales. In: Eustache F, Agniel A, eds. Neuropsychologie clinique des démences: évaluations et prises en charge. Marseille: Solal, 1995:123-36.

88 Grafton ST. PET imaging of human motor performance and learning. In: Boller F, Grafman J, eds. Handbook of neuropsychology. Amsterdam: Elsevier, 1995;10:405-22.

89 Keane MM, Gabrieli JD, Ennema AC, Growdon JH, Corkin $\mathrm{S}$. Evidence for a dissociation between perceptual and conceptual priming in Alzheimer's disease. Behav Neurosci 1991;105:326-42.

90 Keane MM, Gabrieli JD, Growdon JH, Corkin S. Priming in perceptual identification of pseudo-words is normal in Alzheimer's disease. Neuropsychologia 1994;32:343-56. 